\title{
CoolGAL: a Galinstan bathed Be fast neutron production target at the NEPIR facility
} \author{
and Jeffery Wyss ${ }^{2,4}$ \\ ${ }^{1}$ INFN Laboratori Nazionali di Legnaro, vale dell'Università 2, I-35020 Legnaro (Padua), Italy \\ ${ }^{2}$ INFN Padova, via Marzolo 8, I-35131 Padova, Italy \\ ${ }^{3}$ University of Padova, Physics and Astronomy Department, via Marzolo 8, I-35131 Padova, Italy \\ ${ }^{4}$ DICeM, University of Cassino, via Di Biasio 43, I-03043 Cassino (Frosinone), Italy
}

Rogelio Alfonso Barrera ${ }^{1}$, Dario Bisello ${ }^{2}$, Juan Esposito ${ }^{1}$, Pierfrancesco Mastinu ${ }^{1, *}$, Gianfranco Prete ${ }^{1}$, Luca $_{\text {Silvestrin }}{ }^{3}$

\begin{abstract}
In this contribution, we present the CoolGAL fast neutron production target system, to be used in the initial phase of the NEPIR irradiation beamline at the SPES facility, that will be operational in 2022. Initially, NEPIR will be used for shielding studies against fast neutrons for space applications and to investigate neutron-induced single event effects in microelectronic devices and systems. In CoolGAL, the neutron production component, a thick Be cylinder, is immersed in a static bath of Galinstan, a liquid alloy of $\mathrm{Ga}$, In and $\mathrm{Sn}$, contained by an outer water cooled copper cladding. MCNPX calculations indicate that, by using a $1 \mu \mathrm{A}$ current of $70 \mathrm{MeV}$ protons, it can produce a fast neutron energy spectrum that is somewhat flat the 30-65 MeV energy range and with a sharp cut-off at the beam energy. At the standard test point, located $2.6 \mathrm{~m}$ downstream from the source, the beam spot diameter, defined by the peculiar collimation scheme of the initial phase of NEPIR, is $10 \mathrm{~cm}$ and the integral fast neutron flux is $\Phi_{\mathrm{n}}\left(1<\mathrm{E}_{\mathrm{n}}<65 \mathrm{MeV}\right) \sim$ $3 \times 10^{6} \mathrm{n} \mathrm{cm}^{-2} \mathrm{~s}^{-1}$. Using proton beams with two different energies, one can calculate, by subtraction, the effects due to the neutrons in the energy interval defined by the two cut-off values. Preliminary results of ANSYS calculations, for a $1 \mu \mathrm{A}$ proton current of $70 \mathrm{MeV}$ protons $(70 \mathrm{~W})$, show a limited regime temperature $\left(28^{\circ} \mathrm{C}\right)$ of the Be component, capable of ensuring the exceptional safety level required for the operation at SPES. The thermal reliability of CoolGAL is very promising for future developments that require higher proton currents.
\end{abstract}

\section{Introduction: NEPIR}

NEPIR (NEutron and Proton IRradiation facility) is a multidisciplinary project at the new 30-70 $\mathrm{MeV}$ cyclotron of SPES [1] at the INFN National Laboratories of Legnaro (LNL). NEPIR will be built in stages, according to available funds. In the final configuration, it will deliver complementary fast neutron beams: quasi mono-energetic neutron (QMN) beams in the 30-70 $\mathrm{MeV}$ energy range and an intense neutron beam, with a continuous energy distribution in the $1-65 \mathrm{MeV}$ range, similar to that produced by cosmic rays in the atmosphere found at flight altitudes and at sea level. Direct variable energy proton irradiations will be also available to study the effects by protons (e.g. solar protons) too. By using proton-beam energy degraders, the lowest energy of both the QMN and direct protons will be lowered below $30 \mathrm{MeV}$ down to $20 \mathrm{MeV}$, possibly lower.

NEPIR was originally conceived to study the effects of neutrons and protons on electronic components, an important field of both scientific and technological research. The capability of electronic circuits to survive exposure to radiation is essential for many scientific and technological applications in radiation hostile environments: outer space, surface of planets, highenergy physics experiments, nuclear reactors, medical and industrial applications. But the issue radiationassurance of state-of-the-art electronics used in everyday life applications is a growing concern.

The trend towards extreme miniaturization of commercial electronics (not specifically rad-hard) has brought an increased sensitivity to certain kinds of effects induced by neutrons present at sea level. The industrial concern for the effects of these invasive and ubiquitous atmospheric neutrons in vital electronic instrumentation has always been and continues to be found in avionics, but it is an ever growing concern for the fast growing high-reliability ground-based information-technology electronics industry too. At sealevel the fast $(\mathrm{E}>1 \mathrm{MeV})$ neutron flux on earth is $21 \mathrm{n}$ $\mathrm{cm}^{-2} \mathrm{hr}^{-1}$; it is much lower than that at flight altitudes ( 300-400 times), but the number of ground-based

Corresponding author: pierfrancesco.mastinu@lnl.infn.it 
digital electronic devices used in ever-increasing and diverse applications is enormously larger [2][3][4][5][6].

Big European and Japanese industrial groups have shown interest in the NEPIR project at LNL and are waiting for a chance to use the facility. NEPIR will be a precious applied research and test facility also for small/medium high-tech industries, typical of the local and national economy, that develop high-reliability electronic devices and systems for various applications; e.g. information technology, industrial automation, telecommunication, automotive, biomedical, security and military.

NEPIR will also be used to study shielding against fast neutrons and protons. For this purpose, the facility has been partially funded by the MIUR within the SPARE (Space Radiation Shielding) project: a joint INFN, ASI and Centro Fermi collaboration that deals with health risks due to cosmic radiation: a major showstopper for safe space exploration and colonization, where shielding techniques are the only practical countermeasure against radiation. The main goal of the SPARE project is to perform experimental tests, with neutrons and protons, of physical (active and passive shielding materials) and biomedical (hibernation) countermeasures for human space exploration. For novel shielding materials, based on composites and nanomaterials (proprietary, with undisclosed exact composition), or made of complex in-situ available planetary resources, code predictions have high uncertainties or may be completely lacking. Acceleratorbased measurements are in those cases an essential tool to characterize shielding capabilities and to tune models. Compared to the surface of the earth, the fast neutron flux on the surface of the Moon is two orders of magnitude higher, as on the surface of Mars where the spectrum is even harder [7]. The SPARE project includes a test campaign of active and passive shielding materials for fast neutrons at LNL and for protons at the accelerator facility of TIFPA [8].

With the limited SPARE funds, we are building NEPIR-0: a simplified phase- 0 fast neutron irradiation facility, adequate for the SPARE project, but still attractive for academic and electronics industrial users. NEPIR-0 is completely funded and could be completed and commissioned by 2022 and then made available to users.

Indeed the goal of NEPIR-0 is to catalyze further support in order to obtain the necessary funds to upgrade to the final configuration, that would make NEPIR a unique multidisciplinary facility in Europe, very useful for research: applied, industrial and basic.

\section{NEPIR-0}

The floor plan of NEPIR-0 is shown in Figure 1. Its neutron production target is based on a $\sim 30 \mathrm{~mm}$ thick proton stopping $\mathrm{Be}$ target, that produces a continuous energy neutron beam. NEPIR-0 has no independent shielding: it uses instead the shield wall of the SPES facility by placing the Be target inside the conduit that connects the cyclotron hall to the experimental one (section 2.1 and Figure 2). For this reason, the installation of the beam optics for QMN is not possible in this phase of the project.

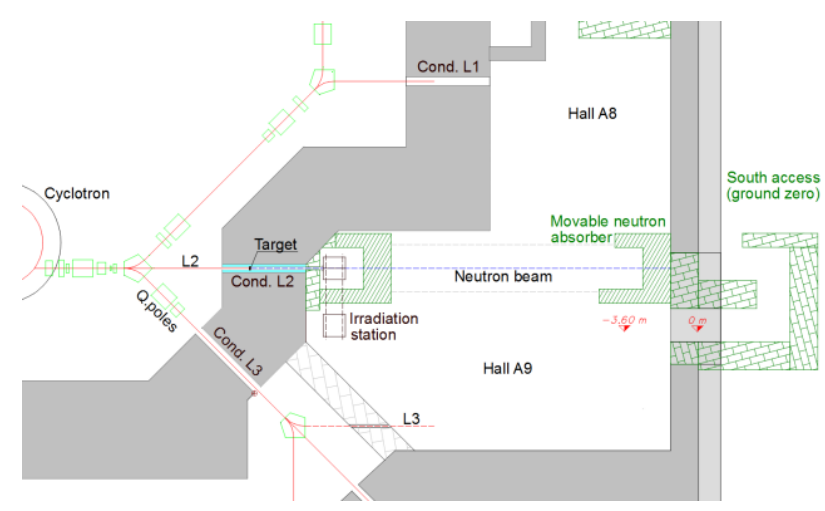

Fig. 1 Floorplan of the L shaped NEPIR experimental area (Halls A9 and A8) and part of the cyclotron hall. The cyclotron is visible on the left. The position of the NEPIR-0 neutron production target system is placed inside the conduit through the $3 \mathrm{~m}$ thick shield wall that separates the cyclotron hall from the experimental hall A9. A movable neutron beam absorber is schematically shown in both open and closed positions.

Because there is not well defined bunker, a low pressure containment system of the activated air of the experimental hall will not be available. For this reason the proton current on the Be target is at most $1 \mu \mathrm{A}$, to ensure that the average activation, diluted by the large ventilated volume of the experimental hall, remains below $1 \mathrm{~Bq} / \mathrm{gr}$.

A movable neutron beam stopper, shown schematically in Figure 1, will reinforce the shielding of the back wall; in the closed position, it will allow users to access the experimental hall A9 while the cyclotron delivers beams to other users; it will also shield against the radiation of the activated beamline materials.

\subsection{The target station and collimation system}

The placement of the Be-based neutron production target inside the conduit is shown in Figure 2.

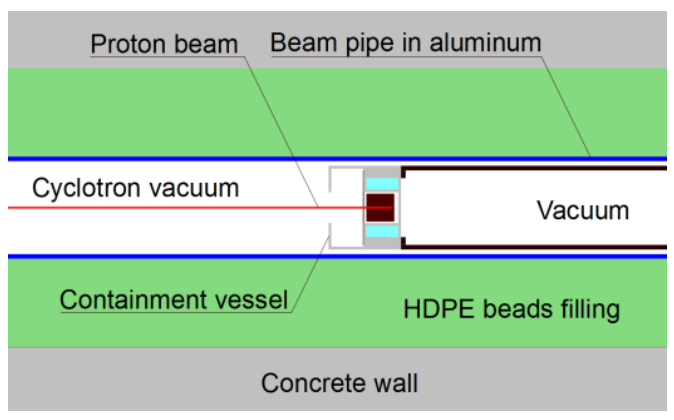

Fig. 2 Schematic representation of the target system inside the conduit through the cyclotron concrete shield wall (grey). In green, the polyethylene filling in the gap between the vacuum line and the wall. A composite material plug (in black) is used to insert the target system and then to hold it place.

The proton vacuum line passes through the conduit in the cyclotron shield wall and is accessible through a 
flange in the experimental hall. The gap between the vacuum pipe and the conduit (30 $\mathrm{cm}$ diameter) is filled with High Density PolyEthylene (HDPE) beads, both to limit neutron propagation to and from the cyclotron hall, and to act as a collimator to define the neutron beam into the experimental area.

The target system is inserted $1 \mathrm{~m}$ inside the conduit, measured from the cyclotron hall. At the end of the conduit, a $50 \mathrm{~cm}$ layer of concrete blocks is added to the cyclotron shield wall to ensure adequate containment of the neutrons produced by the target (visible in Fig. 1). A composite material plug (shown in black in

Fig. 2) is used to insert the target system and its cooling system into the beamline through the flange in hall A9, and to hold it in place. This plug can be evacuated by a dedicated pumping system. At the standard test point, $2.6 \mathrm{~m}$ downstream of the target, the effective diameter of the fast neutron beam is $10 \mathrm{~cm}$.

\subsection{The neutron spectra of NEPIR-0}

Figure 3 shows the expected neutron energy spectra (in the forward direction, with a $3^{\circ}$ semi-angle) produced by the $30 \mathrm{~mm}$ thick $\mathrm{Be}$ neutron production target, for different values of the energy of the impinging protons. Simulations have been performed with the MCNPX code [9].

For a $70 \mathrm{MeV}$ proton current of $\sim 1 \mu \mathrm{A}$, the integral fast neutron flux at the standard test point, $2.6 \mathrm{~m}$ from the target, is $\Phi_{\mathrm{n}}\left(1<\mathrm{E}_{\mathrm{n}}<70 \mathrm{MeV}\right) \sim 3 \times 10^{6} \mathrm{~cm}^{-2} \mathrm{~s}^{-1}$. At the most distant test point, $12.6 \mathrm{~m}$ from the target, the diameter of the neutron beam increases to $50 \mathrm{~cm}$ while the integral neutron flux drops to $\Phi_{\mathrm{n}}\left(1<\mathrm{E}_{\mathrm{n}}<70 \mathrm{MeV}\right) \sim 1.5 \times 10^{5} \mathrm{~cm}^{-2}$ $\mathrm{s}^{-1}$.

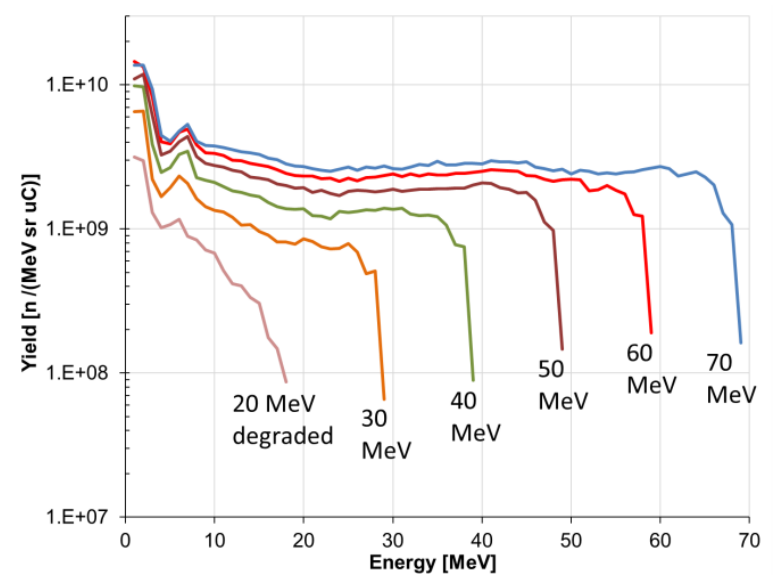

Fig. 3. Simulated (MCNPX) fast neutron spectra in the forward direction for different proton beam energies.

For proton energies greater than $30 \mathrm{MeV}$, the distributions are somewhat flat, up to a sharp cutoff value close to the energy of the proton beam. This feature will be exploited to give NEPIR-0 pseudo-QMN capabilities: by irradiating a sample with neutrons produced using proton beams with two different energies, one can calculate, by subtraction of the renormalized distributions, the effects due to neutrons in the energy interval defined by the two cut-off values. An example is shown in Figure 4, where a neutron spectrum obtained by a $60 \mathrm{MeV}$ proton beam is normalized and subtracted from the spectrum by a $70 \mathrm{MeV}$ proton beam. The effective neutron spectrum corresponds to a box-like distribution in the 58-68 MeV (FWHM) energy interval, with an integral flux of $\sim 2.8 \times 10^{5} \mathrm{~cm}^{-2} \mathrm{~s}^{-1}$.

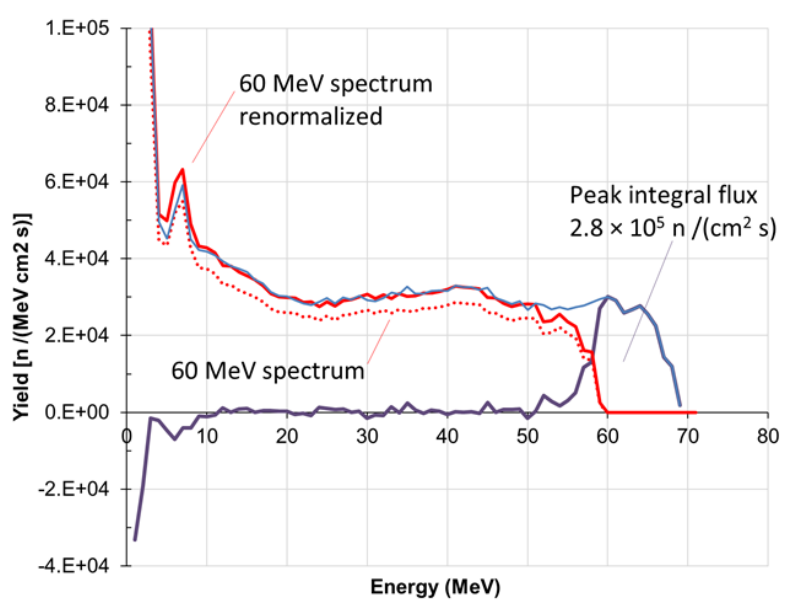

Fig. 4. The effective neutron spectrum obtained by subtraction of neutron spectra generated by $60 \mathrm{MeV}$ (renormalized) and 70 $\mathrm{MeV}$ proton beams.

\subsection{The CoolGAL target system of NEPIR-0}

At NEPIR-0 the $70 \mathrm{MeV}$ proton current is limited to 1 $\mu \mathrm{A}$. For this reason we initially considered a simple low power $(<100 \mathrm{~W})$ air-cooled Be cylinder $(\varnothing=30 \mathrm{~mm}$, thickness $=30 \mathrm{~mm}$ ) with an aluminum cladding and air cooling fins. However, any cladding must be designed carefully to account for thermal expansions all while maintaining good contact. In addition Be is well known to be problematic, as proton irradiation eventually causes swelling, the formation of bubbles and defoliation, with the risk of contaminating the cyclotron beam line. It is of paramount importance that the target be free to expand and that any debris be contained.

For these reasons we are proposing CoolGAL: a more sophisticated target system (Fig. 5 and 6). In CoolGAL, the Be cylinder is immersed in a static bath of a liquid metal eutectic alloy of Gallium, Indium and Tin (Sn), commercially known as Galinstan [10]. The liquid metal, contained by a copper cladding, ensures a good thermal contact with the $\mathrm{Be}$ and the internal surface of the $\mathrm{Cu}$ cladding, Au-coated to ensure tolerance to Galinstan. A 25 micron thick, robust havar [11] membrane separates the liquid metal from the beamline vacuum; this entrance window is cooled efficiently as it is in contact with the liquid metal. The outer surface of the cladding and a matching copper outer layer define a cooling circuit for a flowing coolant (water). To improve heat exchange, the outer surface of the cladding has cooling fins. The water flow in the enclosed circuit is ensured by input and output lines connected to a chiller in the experimental area.

The cladding is evacuated trough the reservoir tank prior to be filled with liquid metal and a pressure gauge is used to monitor the vacuum condition on top of the 
liquid. Any degassing reaction products that may leave the Be target during and after an irradiation would be confined in this tank, thereby keeping these contaminants from diffusing towards the cyclotron. If a threshold pressure value is exceeded, the proton beam is stopped, the target system extracted and inspected and the outgassed material disposed of in a proper way through a vent valve.

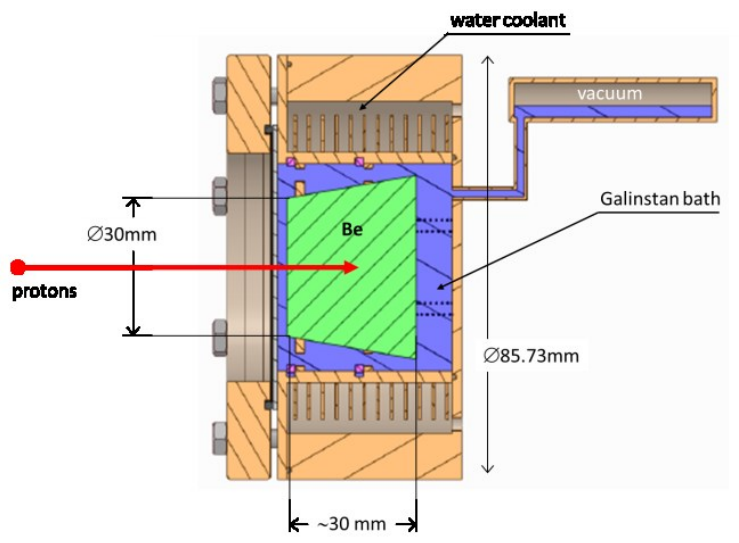

Fig. 5. Conceptual representation of the CoolGAL target system. The proton beam comes from the left, crosses a havar 25 micron thick window (visible in Fig.6) and a $1 \mathrm{~mm}$ thick liquid layer before it is stopped by the thick Be component (yellow). The Be is immersed in a Galinstan bath (purple) contained by a copper cladding.

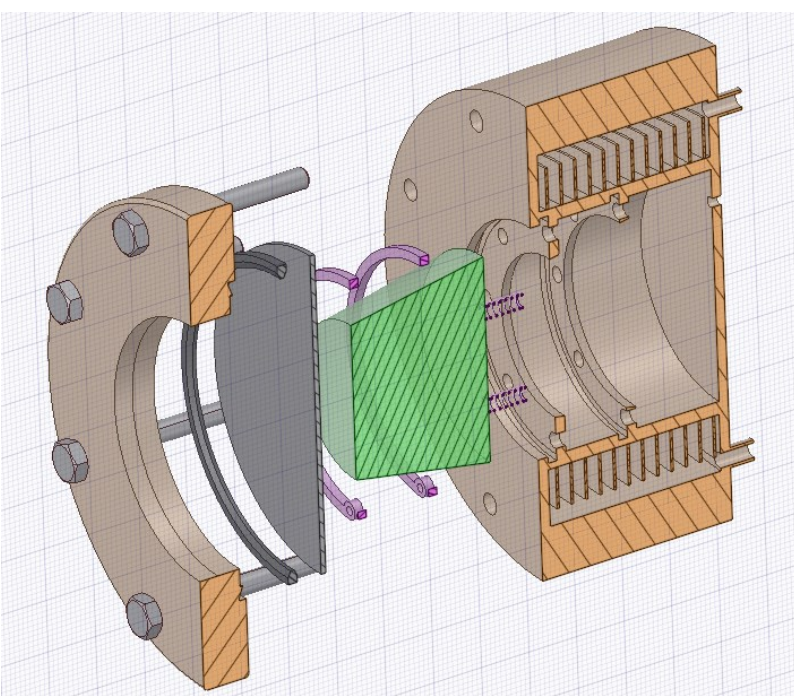

Fig. 6. Exploded view of the CoolGAL ANSYS model. The finned outer surface of the cladding and a matching copper outer layer define a cooling circuit. The surface of the cladding in contact with the coolant (flowing water) has cooling fins. Forced air cooling is not viable.

The working scheme of the Be target is shown in Fig. 7 , together with the setup to fill it with liquid metal. The procedure to fill the target with the liquid metal is made up of four steps:

1. At the beginning valve $\mathrm{V} 3$ is closed and a vacuum pump is used to evacuate the target, down to a pressure of the order of $10^{-6} \mathrm{mbar}$. The pressure is monitored with the Pirani gauge located on of the expansion vessel. In this condition the pressure difference across the havar window is 1 atm, the thickness of the window is selected to enable it to withstand the provided stress. The slow pumping down time avoids any impulsive force.

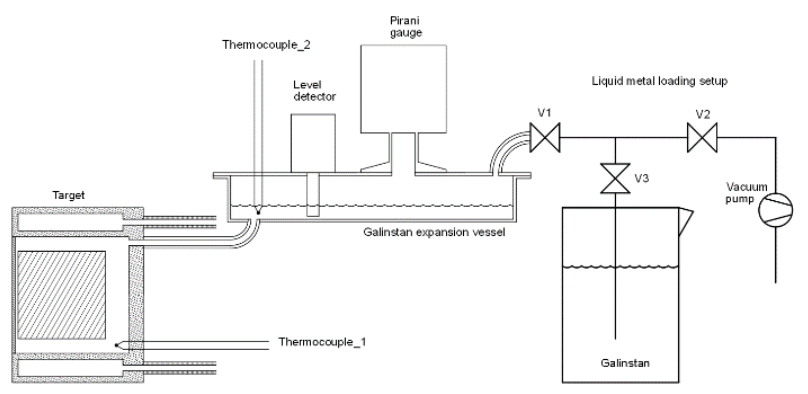

Fig. 7 Logic working scheme of the CoolGAL target system, including the setup to load it with Galinstan and provide the vacuum in the expansion vessel.

2. Valve V2 is closed to isolate the system from the outside environment.

3. Valve V1 is opened allowing the suction of the required amount of liquid metal in the evacuated expansion vessel and target cladding. By gravity, the liquid will completely fill the cladding, wetting all of the surface of the Be neutron converter and all the inner surface of the cladding. Also a small fraction of the inner volume of the expansion vessel will be filled with Galinstan, the rest will remain evacuated.

4. Valve V1 is closed, isolating the target system.

At this point the loading setup can be removed and the target installed. The target assembly is provided of four sensors to guarantee its safety both during working time and during rest time. Two temperature sensors (thermocouples) are used to measure the Galinstan temperature inside the cladding and inside the expansion vessel. A Pirani gauge will continuously monitor the vacuum in the expansion vessel to detect possible release of gasses (potentially radioactive) from the $\mathrm{Be}$ component. A level detector will be used to ensure the stability of the level of the cooling liquid.

In normal conditions, the pressure difference across the havar window will be only the small hydrostatic pressure provided by the Galinstan (much smaller than 1 atm). In case of rupture of the havar, the Galinstan will gently pour out and will be collected by a cylindrical containment vessel placed in front of the havar window (visible in Figure 2).

\subsection{ANSYS calculations of CoolGAL}

The final design of the target is currently under way and near completion.

Preliminary thermo-mechanical ANSYS [12] calculations of the minimal model show that, with a 1 $\mu \mathrm{A}$ proton current of $70 \mathrm{MeV}$ protons $(70 \mathrm{~W})$, forced air cooling is not a viable solution; flowing water as coolant is, instead, very promising. 
ANSYS calculated regime temperature distributions in the Be and the Galinstan at $70 \mathrm{~W}$ for water cooling are shown in Figure 9 for a simple model: the power deposition of the beam is described as a cylinder $1 \mathrm{~cm}$ in diameter and the natural convection in the Galinstan is simulated by including buoyancy. The calculations show that there is a tendency of the temperature to be higher at the top of the Galinstan due to the natural movement caused by the density effect. This effect is evident in Figure 10 that shows the temperature distribution for 700 $\mathrm{W}$; it is not apparent at $70 \mathrm{~W}$, as the temperature differences are too small.

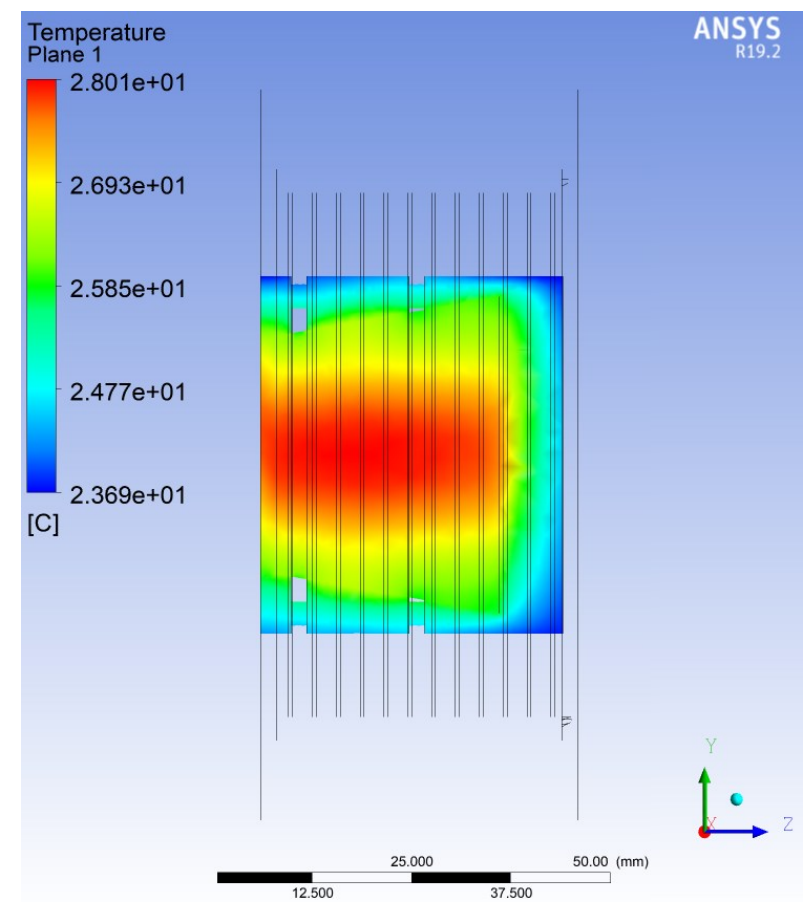

Fig. 9. Temperature map of the $\mathrm{Be}$ and Galinstan of a preliminary ANSYS model of CoolGal with water coolant for a $1 \mu \mathrm{A}$ current of $70 \mathrm{MeV}$ protons $(70 \mathrm{~W})$. The maximum temperature of the $\mathrm{Be}$ component is $28^{\circ} \mathrm{C}$.

The very low maximum temperature $\left(28^{\circ} \mathrm{C}\right)$ of the Be component at $70 \mathrm{~W}$ guarantees the exceptional safety level required for a target located so close to the cyclotron (distance $<8 \mathrm{~m}$ ), that no effective counter measures can be implemented to prevent contaminations in case of catastrophic failure.

The thermal reliability of CoolGAL with the present design stage is also very promising for future developments requiring higher proton currents: Figure 11 shows the maximum temperature of the $\mathrm{Be}$ component as a function of power. In the present design, there are only convective currents in the Galinstan liquid; to improve performance further, we plan to consider a flowing Galinstan circuit.

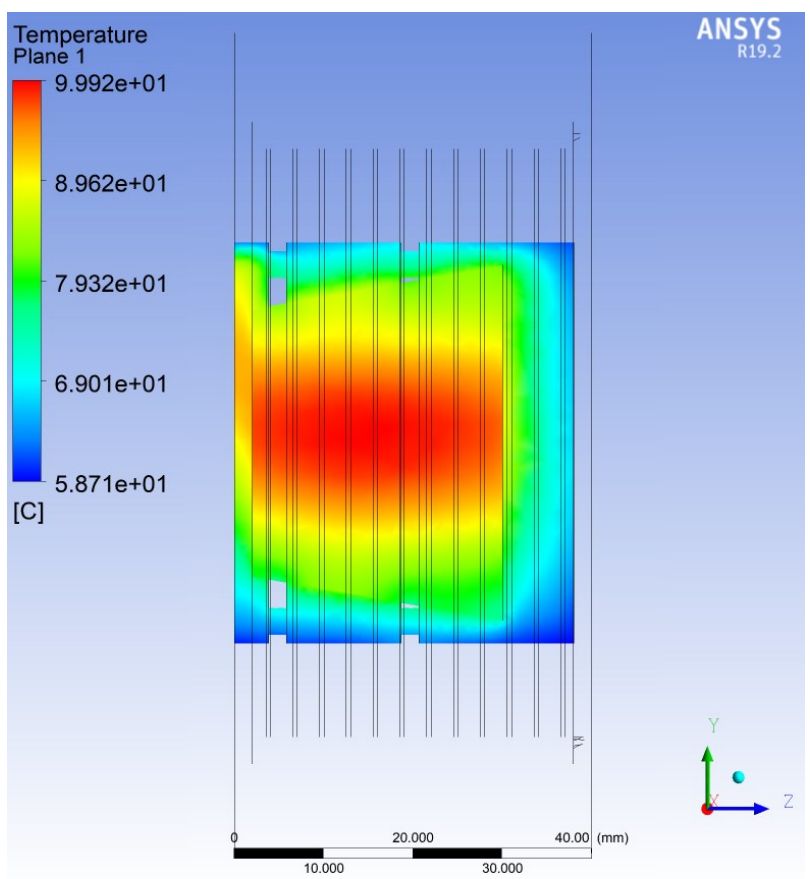

Fig. 10. Temperature map of the Be and Galinstan of a preliminary ANSYS model of CoolGal with water coolant for $700 \mathrm{~W}(10 \mu \mathrm{A}$ current of $70 \mathrm{MeV}$ protons $)$. The maximum temperature of the $\mathrm{Be}$ component is $100^{\circ} \mathrm{C}$.

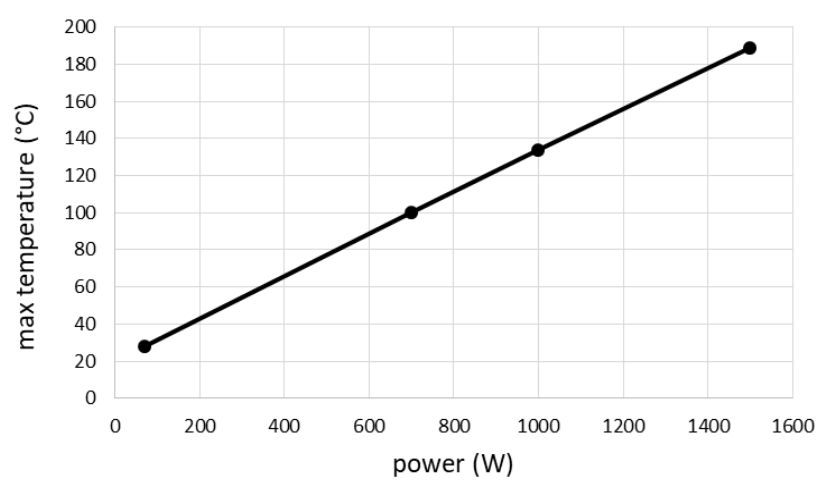

Fig. 11. Summary of the maximum temperature of the Be component versus beam power of the ANSYS model of CoolGAL using water coolant flowing at $\mathrm{v}=2 \mathrm{~m} / \mathrm{s}$ with an input temperature of $20^{\circ} \mathrm{C}$.

\section{Conclusions}

In summary, the advantages of the liquid metal bath in CoolGAL are:

- it allows thermal dilatation of the beryllium;

- it ensures thermal contact and heat transfer to the copper cladding for conduction;

- thanks to natural convection, it distributes the heat on the whole surface of the cladding;

- it cools down the havar window;

- it contains any debris of beryllium if/when blistering occurs;

- it is viable for higher power applications.

Our goal is to commission the NEPIR-0 facility by the end of 2020-beginning 2021 and to open it to users from academia and especially industry in 2022.

The rapid implementation and success of NEPIR-0 with users, especially industrial ones, will catalyze the 
upgrade to the final NEPIR configuration, the first step of which will be the construction of a dedicated independent bunker and air containment system, followed by the installation of the beam optics allowing the facility to deliver fast QMN beams, while maintaining and improving the capability of the Be target to provide a high flux of continuous energy neutrons.

\section{References}

1. G. Prete et al., The SPES project at the INFNLaboratori Nazionali di Legnaro, EPJ Web of Conferences 66, 11030 (2014)

2. M. S. Gordon et al., Measurement of the flux and energy spectrum of cosmic-ray induced neutrons on the ground, IEEE Trans. Nucl. Sci. V. 51 , I. 6 (2004)

3. T. Nakamura, M. Baba, E. Ibe and Y. Yahigi, Terrestrial Neutron-Induced Soft Errors in Advanced Memory Devices (World Scientific Publishing Co. Pte. Ltd, 2008)

4. JESD89A, Measurement and Reporting of Alpha Particle and Terrestrial Cosmic Ray-Induced Soft Errors in Semiconductor Devices, Oct. 2006.

5. M. Bagatin, S. Gerardin, Soft errors in floating gate memory cells: A review, Microelectron. Reliab. V. 55 I. 1, 2015, P. 24-30

6. W. Yang et al., Atmospheric neutron single event effect test on Xilinx $28 \mathrm{~nm}$ system on chip at CSNSBL09, Microelectron. Reliab. V. 99, 2019, P. 119124

7. J.W. Wilson et al., Deep space environments for human exploration, Advances in Space Research 34 (2004) 1281-1287

8. F. Tommasino et al., Proton beam characterization in the experimental room of the Trento Proton Therapy facility, NIM A, V 869, 2017, P. 15-20

9. D. B. Pelowitz, M. R. James, G. W. McKinney, J. W. Durkee et al., MCNPX 2.7.A Extensions (LANL, 2008).

10. https://en.wikipedia.org/wiki/Galinstan

11. https://en.wikipedia.org/wiki/Havar_(alloy)

12. ANSYS $®$ Academic Research, Release 19.2 